\title{
The Where, What and Why of the Developing Renal Stroma
}

\author{
Luise A. Cullen-McEwen Georgina Caruana John F. Bertram \\ Department of Anatomy and Cell Biology, School of Biomedical Sciences, Faculty of Medicine, Nursing and \\ Health Sciences, Monash University, Clayton, Australia
}

\section{Key Words}

Stroma - Interstitium - Branching morphogenesis • Nephrogenesis

\begin{abstract}
In recent years, a great deal has been learnt about the molecular regulation of kidney development. While most research has focused on the molecular regulation of ureteric branching morphogenesis and nephron formation, significant insights into the definition and functions of the renal stroma have emerged. Many molecules expressed in the developing renal stroma are now known to play significant regulatory roles in kidney development. However, the term 'renal stroma' continues to have different meanings to different researchers. This review clarifies this situation and defines the derivation, location and functions of the stroma in the developing metanephros.
\end{abstract}

Copyright ( 2005 S. Karger AG, Basel

\section{Introduction}

The permanent kidney (metanephros) develops when the ureteric bud invades a mesenchymal tissue known as the metanephric blastema. It is the complex interactions between these tissues that result in the ureteric bud, undergoing branching morphogenesis or repetitive epi- thelial cleft and bud formation, which ultimately creates the complex three-dimensional branching structure of the collecting duct system. Simultaneously, the tips of the branching ureteric tree, as they branch towards the periphery of the developing kidney known as the nephrogenic zone, induce the formation of nephrons. These reciprocal interactions continue throughout development and the first few days of life to form approximately 12,000 nephrons per kidney in the normal mouse.

In recent years, much progress has been made in understanding the molecular and cellular mechanisms during metanephric development that regulate branching morphogenesis and nephron formation [1-7]. However, the subpopulation of the renal stroma and its role during metanephric development has become an exciting area of study for researchers in the field. This review seeks to clarify and define the derivation, location and functions of the stroma during metanephric development.

\section{Metanephric Development and the Origin of Renal Stroma}

The metanephros is classically described as being derived from two embryonic precursor tissues, the ureteric duct epithelium and the metanephric blastema (also known as metanephric mesenchyme), both of which develop from intermediate mesoderm. The metanephric blastema is comprised of a non-homogeneous population

\begin{tabular}{ll}
\hline KARGER & ( ) 2005 S. Karger AG, Basel \\
1660-2129/05/0991-0001\$22.00/0 \\
$\begin{array}{l}\text { Fax +4161306 12 34 } \\
\begin{array}{l}\text { E-Mail karger@karger.ch } \\
\text { www.karger.com }\end{array}\end{array}$ & $\begin{array}{l}\text { Accessible online at: } \\
\text { www.karger.com/nee }\end{array}$
\end{tabular}

Prof. John F. Bertram

Department of Anatomy and Cell Biology, School of Biomedical Sciences Faculty of Medicine, Nursing and Health Sciences, Monash University

Clayton, Vic. 3800 (Australia)

Tel. +61 39905 2751, Fax +61 39905 2766, E-Mail john.bertram@med.monash.edu.au 
of cells that sends signals to the Wolffian duct, which in response gives rise to the ureteric bud. The ureteric bud grows towards and penetrates the metanephric blastema, and a series of reciprocal inductions between the ureteric bud and the metanephric blastema result in the formation of the permanent kidney. The term 'induction' refers to 'the stimulation of a specific developmental pathway in one group of cells (the responding tissue) by a closely approximated group of cells (the inducing tissue)' [8]. Induction of the metanephric blastema is a two-step process. The first step involves differentiation of the metanephric blastema into 'blastemal cells'. These are small unpolarized cells with little cytoplasm and are often termed 'stem' or 'progenitor' cells. However, the fundamental questions asked by kidney development researchers today revolve around how many cell lineages are in the metanephric blastema before and after being induced by the ureteric bud. Is the metanephric blastema a mass of multipotent stem cells, which, with the right cues, develop into the many cell types found in the metanephros? Or does the metanephric blastema contain a variety of predetermined precursor cell lineages? Or does it contain both? As these questions remain to be answered, Sariola et al. [9] have alternatively termed these mesenchymal cells as blastemal cells, to describe them by a neutral word in terms of their origin.

With the first stage of induction by the ureteric bud, blastemal cells are rescued from apoptosis and induced to proliferate $[10,11]$. The second stage of induction occurs when the ureteric bud has invaded the metanephric blastema, and the blastemal cells differentiate along different developmental pathways with the formation of the 'cap' (described below) and renal stroma. However, although without ureteric bud invasion metanephric development ceases, it is not known whether it is the ureteric bud that instructs these progenitors to differentiate along these different pathways or whether they are already differentiated prior to the invasion of the ureteric bud.

To date, the origin of the renal stroma is unknown. These cells have been traditionally described as developing from cells of the metanephric blastema that were not induced to condense and epithelialize [12]. However, these cells are now often described as developing from a separate cell lineage within the metanephric blastema [13, 14], and furthermore there is also a hypothesis that these cells may be of neural crest origin $[15,16]$. Stromal cells in the mouse kidney express the neural crest marker, disialoganglioside GD3 [17] and neurofilament light and medium proteins [16]. In addition, mouse explant cultures contain neurons [15], and early transplantation studies have shown that when neural crest from the quail is transplanted into chick embryos, quail neural crest cells can be found in the chick mesonephric stroma [18]. Furthermore, labelled neural crest cells of the chick have been found in the metanephros [19].

Nephron formation begins when blastemal cells directly associated with the tips of the ureteric tree condense, forming a mesenchymal cap. Cap cells induce the ureteric bud to lengthen and bifurcate as it extends. Once the tips reach the periphery at approximately embryonic day 13 (E13) in the mouse, blastemal cells are located as a thin rind in the outermost cortex. A small percentage of cap cells located at the base of the extending tip are induced to form a mesenchymal condensate, which undergoes mesenchyme to epithelial transition (MET) and ultimately differentiates into a nephron. In response, cap cells in direct contact with the ureteric bud tips stimulate the ureteric epithelial cells to proliferate and the duct to branch dichotomously, ultimately forming the collecting duct system.

\section{Renal Stroma: Where Is It and What Is It?}

In the mouse, metanephric development begins at approximately E10.5 with formation of the ureteric bud. By E11, the ureteric bud has invaded the surrounding blastema (mesenchyme), and the cells are induced to survive and proliferate. At this stage, the blastema consists of a group of morphologically similar cells (fig. 1a). The ureteric bud then induces the blastemal cells to condense around the ureteric bud tip (fig. 1b), and two regions of the metanephric mesenchyme can be morphologically distinguished: (1) the region surrounding the ureteric bud tip is condensing cap mesenchymal cells which are Pax 2 positive and will ultimately form nephrons, and (2) the region peripheral to the cap mesenchyme is a looser domain of cells which express the winged helix transcription factor Foxd 1 (formerly BF2 - [20]) (fig. 1b). These latter cells are the presumptive stromal progenitor cells [20]. Within the nephrogenic zone is a 3rd population of cells (fig. 1b) which are Foxd 1 negative and appear smaller and rounder than Foxd1-positive stromal precursor cells. Foxd1negative cells may be mesenchymal stem or progenitor cells [20]. By E11.5, the condensing mesenchyme has induced the ureteric bud to divide, forming a T-shaped tubule, and mesenchyme condenses around both ureteric tips (fig. 1c). By E12-13, when arborization of the ureteric tree has begun and nephron induction is taking place, in addition to the stromal progenitor cells found in the neph- 
Fig. 1. Development of the renal stroma/ interstitium. At approximately E10.5 in the mouse, the metanephric blastema induces the formation of the ureteric bud from the Wolffian duct. By E11, the ureteric bud has invaded the surrounding mesenchyme (blastema), and the mesenchyme is induced to survive and proliferate. At this stage, the blastema consists of a group of morphologically similar mesenchymal cells (a). The ureteric bud then induces some blastemal cells to condense around the ureteric bud tip (b). At this stage, two regions of the metanephric mesenchyme can be morphologically distinguished. Surrounding the ureteric bud tip are condensing mesenchymal, Pax2-positive 'cap cells', which will ultimately form nephrons (large round cells). Peripheral to this mesenchyme is a looser domain of cells, the stromal progenitor cells which express Foxd 1 (spindle-shaped cells) (b). In addition to these two regions, there are small round cells, which are Foxd1 negative and have been suggested to be the renal stem cell population. These are found in the nephrogenic zone and tend to concentrate in areas between ureteric branch tips [20]. By E11.5, the condensing mesenchyme has induced the ureteric bud to divide, forming a T-shaped tubule and mesenchyme condenses, forming caps around both ureteric tips (c). By E1213, when arborization of the ureteric tree has begun and nephron induction is taking place, in addition to the stromal progenitor cells found in the nephrogenic zone, stromal cells are also found surrounding the ureteric bud branches and induced nephrons, in what is often referred to as primary renal interstitium (grey area - cells are not shown for purposes of simplicity) (d). These stromal cells express GD3, tenascin and Foxd1. By late gestation, two distinct stromal populations are defined, the cortical stroma (light grey) found intercalated between induced nephrons towards the periphery, and the medullary stroma (dark grey), while the presumed stromal progenitor cells remain in the nephrogenic zone (white). Stromal cells are not shown for purposes of simplicity (e).

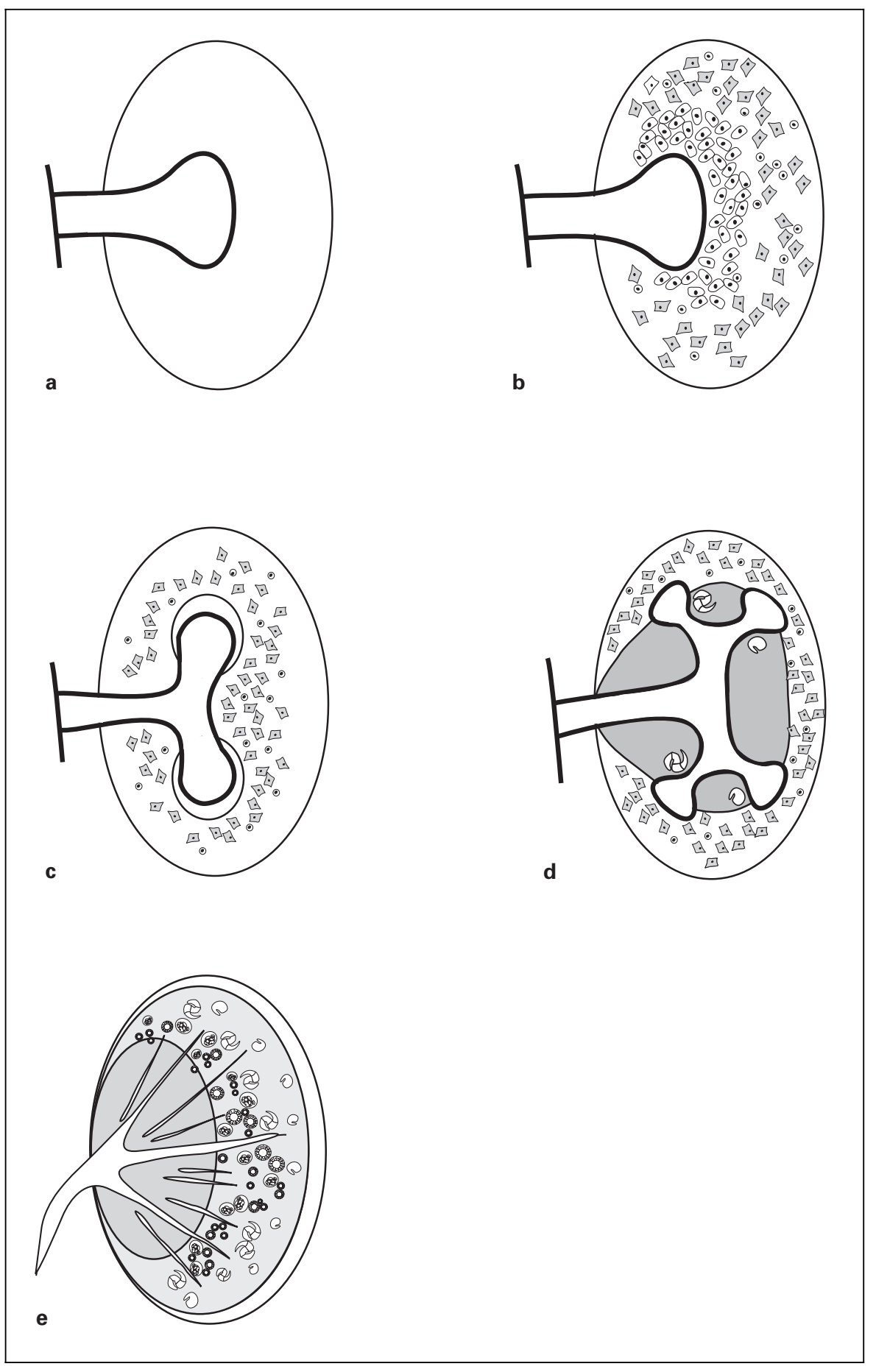

rogenic zone, stromal cells are found surrounding ureteric bud branches and induced nephrons. These stromal cells are arranged in layers and are often referred to as primary renal interstitium [21] (fig. 1d). The primary interstitium, or 'clear cell type renal stroma' [9], is characterized by large fibroblastic spindle cells, with extensive 'clear' cytoplasm separated by large amounts of extracellular matrix (ECM) consisting of mesenchymal proteins such as collagens, tenascin and fibronection, and integral basement membrane glycoproteins (type IV collagen, laminin and 
proteoglycans) [22]. The primary interstitium is morphologically recognized soon after nephron induction [5]. These stromal cells exclusively express the cell surface glycolipid disialoganglioside GD3 [17], tenascin [12] and Foxd1 [20]. By late gestation, the clear cell type renal stroma is abundant, and as nephron tubules develop and the loops of Henle extend, a secondary interstitium is defined with two distinct stromal populations, the cortical stroma, found intercalated between induced nephrons towards the periphery, and the medullary stroma [23-24] (fig. 1e). Evidence for molecular differences between these two stromal populations was first provided by Mendelsohn et al. [25], and will be discussed later. A central core of primary interstitium remains within the outer medulla and the cortical medullary rays until tubular growth and positioning is complete [21].

The stroma of the developing kidney, in broad terms, has traditionally been described as the supportive framework around the developing nephrons and collecting duct system and is said to be made up of interstitial cells, which synthezise and secrete the surrounding ECM and growth factors, which are essential to support development of the kidney. However, specific roles for the stroma in the regulation of both ureteric branching morphogenesis and nephron formation are now emerging.

By birth, many of the medullary stromal cells have undergone apoptosis, and the space they once occupied is filled by developing loops of Henle [14]. Once growth and differentiation of the kidney are complete, the primary interstitium differentiates into a diverse adult interstitium. The interstitium constitutes approximately $7 \%$ of the cortical volume in the rat kidney [26]. Three of the 7\% represent interstitial cells, while the remaining $4 \%$ represent the ECM [27]. The cortical interstitium in the adult kidney is comprised of fibroblasts and lymphocyte-like cells. The ECM consists of collagen fibrils, proteoglycans, glycoproteins and interstitial fluid [28-30]. Medullary interstitium contains three cell types: prominent lipidladen interstitial cells, lymphocyte-like cells and pericytes.

\section{Role of the Stroma in Metanephric Development}

In recent years, we have learned that the stroma provides much more than an inert structural support for the developing kidney. Indeed, recent findings suggest the stroma partially regulates epithelialization of the metanephric mesenchyme (MET) and ureteric branching.

\section{Role of the Stroma in Ureteric Branching Morphogenesis}

Retinoic acid receptors $\alpha$ and $\beta 2$ (RAR $\alpha$ and RAR $\beta 2$ ) are nuclear receptors involved in vitamin A signalling. At E12, both RAR $\alpha$ and RAR $\beta 2$ are co-expressed with Foxd1 by peripheral stromal progenitor cells. By E14, both receptors are expressed in the nephrogenic zone, intercalated between induced nephrons and in the medullary primary interstitium $[25,31]$. Single RAR $\alpha$ and RAR $\beta 2$ knockout mice show no renal phenotype [32-35]. However, double-mutant mice exhibit small kidneys, reduced nephron number, impaired collecting duct development and no nephrogenic zone at birth [25]. The stromal cells are abnormally located in a thick peripheral zone devoid of ureteric buds, and the stromal population normally interspersed between induced nephrons is absent. At E12, expression of the product of the proto-oncogene c-RET (RET) is almost undetectable in ureteric epithelial tips of the double mutants [35]. This phenotype suggests that stromal cells may signal the ureteric bud to maintain RET expression. Subsequent studies found that restoring RET expression in the ureteric bud of the double mutant rescues branching and stromal cell patterning [31]. This indicates that a signalling loop exists between ureteric bud cells and stromal cells, whereby RARs induce the stromal cells to secrete signals which control RET expression in the ureteric bud, and therefore branching morphogenesis, and in turn adequate RET expression regulates normal stromal cell patterning.

Retinaldehyde dehydrogenase 2 (Raldh2) is the enzyme that converts retinal (vitamin A metabolite) to retinoic acid, which is then able to bind to the RARs. At E12, Raldh2 is found in stromal progenitor cells in the nephrogenic zone, similar to Foxd1 and RAR $\beta 2$. By E14, when the primary interstitium is distinct, Raldh 2 remains only in nephrogenic zone stroma. Selective expression of Raldh2 in the nephrogenic zone restricts retinoic acid synthesis to the periphery, limiting signalling through RARs to the domain where RET is expressed in ureteric bud tips [31].

Fibroblast growth factor 7 (FGF7) modulates ureteric bud growth and the number of nephrons formed in vitro [36]. FGF7 is expressed in renal stromal cells surrounding the growing ureteric bud [37], while its high affinity receptor FGFR2 is expressed by the ureteric epithelium [38]. Kidneys of FGF7 knockout mice contain fewer nephrons and have attenuated branching morphogenesis [36]. In vitro, increased FGF7 levels enhance ureteric bud growth and nephron development [36, 39]. However, FGF7 receptor expression in the ureteric bud indicates that the 
increase in nephron number is likely to be an indirect result of increased branching [36]. Furthermore, the fact that FGF7 can also stimulate growth of isolated ureteric buds further supports the idea that FGF7 produced by renal stromal cells is a growth factor for the ureteric bud.

Bone morphogenetic protein 4 (BMP4) is expressed in stromal cells immediately surrounding the Wolffian duct and ureteric bud, but not around the tips, and its receptors are expressed in the Wolffian duct and ureteric epithelium [40]. BMP4 inhibits budding from inappropriate sites along the Wolffian duct by antagonizing inductive signals sent from the metanephric mesenchyme, as shown by inhibition of Wnt11 expression, a target of GDNF. Similarly, BMP4 diminishes the number of ureteric branches and alters the branching pattern in vitro [40].

\section{Role of Stromal Cells in Nephrogenesis}

Recent evidence suggests that the renal stroma plays a role in the regulation of MET, although this role remains unclear. One of the most important steps in identifying cells of the renal stroma and their role in kidney development was the identification of Foxd 1 and the production of Foxd1 knockout mice. As previously mentioned, Foxd1 is expressed just after invasion of the ureteric bud by a ring of mesenchymal cells surrounding induced Pax2-expressing mesenchymal cells of the nephrogenic zone [20]. These cells are often termed stromal precursor or progenitor cells. At later stages of development (E14+), as the two populations of the renal stroma emerge, Foxd 1 is expressed in both the cortical stroma and in lower levels in the medullary stroma. However, those mesenchymal cells nearest to the ureteric tips, which are destined to undergo MET, never express Foxd1. Homozygous Foxd1 null mutant mice are born with hypoplastic kidneys, with reportedly only $7 \%$ of the number of nephrons of wildtype littermates [20]. Furthermore, Foxd1 mutant kidneys at E14.5 show reduced ureteric bud branching with elongated branches, unrestricted RET expression (that is, not repressed in the stalks of the ureteric branches), ectopic mesenchymal condensates in the medullary region of the kidney, hyperproliferation of mesenchymal cell condensates and inhibited MET [20]. These results demonstrate that stromal cells expressing Foxd1 regulate the synthesis and secretion of signalling molecules which are not only necessary for the appropriate development of the ureteric duct but also for MET.

Recently, two other transcription factors expressed in the stroma, Pbx-1 and Pod-1, were shown to be involved in the regulation of MET [41-42]. Pbx-1 is a three-aminoacid loop extension (TALE) homeodomain transcription factor, and Pod-1 a basic helix-loop-helix transcription factor. Pbx-1 and Pod-1 are initially expressed in stromal progenitor (Foxd1-positive) cells. At E14.5, expression of Pod-1 is seen in 'spindle-shaped' primary interstitial cells (Foxd1 positive, tenascin positive) at the cortical medullary junction and later in medullary interstitial cells. These Pod-1-expressing cells differentiate into the peritubular interstitial cells of the cortex and medulla and pericytes in the adult kidney. Unlike Foxd1, both factors are also expressed in condensing mesenchyme about to undergo MET, with expression being down-regulated as epithelialization proceeds. Both Pbx-1 and Pod-1 knockout mice display similar renal phenotypes as Foxd1 knockout mice, namely expansion of condensed mesenchyme around ureteric tips and delayed MET resulting in a nephron deficit. Ureteric branching is reduced with crowding of branches seen, possibly due to a shortening in branch elongation resulting in a reduction in tips in the nephrogenic zone. This phenotype may be explained by the ectopic expression of RET in the ureteric epithelium when either Pbx-1 or Pod-1 is lost. In Pod-1 nulls, the spindleshaped cells of the primary interstitium are missing, and as a result the renal medulla does not form and the neonatal kidney lacks peritubular interstitial cells [43]. Given that Pod-1 and Pbx-1 are expressed in both condensing metanephric mesenchyme and stromal cells, it was difficult to determine whether the defects seen in MET and ureteric branching in the Pod-1 and Pbx-1 mutants were due to the loss of these factors in the metanephric mesenchyme and/or stroma. In the case of Pod-1, this issue was resolved via the analysis of chimeric mice generated using Pod-1 null ES cells aggregated to ubiquitous GFP expressing embryos [43]. These mice demonstrated that Pod-1mutant cells were able to contribute to the early condensed mesenchyme. However, all spindle-shaped cells and cells of the primary interstitium were derived entirely from wild-type cells. In addition, it was demonstrated that when wild-type cells contributed to the renal interstitial cells, the delay in glomerulogenesis was restored. These results demonstrated that Pod-1 expression in the stroma and not in the condensed metanephric mesenchyme was required for normal nephrogenesis to occur. The similarities in the phenotypes of Foxd1, Pod-1 and Pbx-1 mutants suggest the stroma plays an important regulatory role in nephrogenesis.

Further support for the role of the renal stroma in MET was provided by Yang et al. [44] who showed that culture of isolated stroma-free epithelial progenitors (distinct cellular clusters in the metanephric mesenchyme; see Barasch et al. [45]) with LIF resulted in the formation of 
nephrons. Yang et al. [44] concluded that renal stroma is not critical for MET in vitro. Surprisingly, the number of nephrons produced was greater than in metanephric mesenchyme containing stromal cells. In addition, treatment of these epithelial progenitors with both LIF and proteins secreted by a renal stromal cell line (LacZ-positive BF2/ Foxd1-positive cells [20]) abolished glomerulogenesis but stimulated tubulogenesis. This study showed that nephron epithelial progenitor cells in the metanephric mesenchyme are the target of factors produced by the ureteric bud as well as the renal stroma. Furthermore, the nephron epithelial cell type formed (glomerular or tubular) is regulated by the renal stroma, as the formation of glomeruli is inhibited by renal stromal cells [44].

BMP7 can also act as a survival factor for isolated metanephric mesenchyme in culture [46-48]. However, these cells fail to develop into nephrons even when inducing tissues are present. Instead, these cells display stromal characteristics (expression of Foxd1), suggesting BMP7 may direct mesenchymal cells into stromagenic rather than nephrogenic differentiation.

EGF is thought to act as a survival factor for stroma. Weller et al. [49] analyzed whether growth factors alone can induce isolated metanephric mesenchyme to differentiate into epithelium or interstitium and therefore prevent apoptosis. EGF stimulated expansion of the stromal cell compartment in isolated mesenchyme and when mesenchyme was co-cultured with ureteric epithelium and spinal cord. However, cells were defined as stromal cells based only on their appearance. The expansion of the stromal compartment in response to EGF occurred at the partial expense of epithelial cells. The data of Weller et al. [49] suggest the presence of EGF receptors during metanephric development. However, although the EGF receptor is present in the kidney during development [50], EGF is present only postnatally $[51,52]$. However, transforming growth factor-alpha (TGF $\alpha$ ), a member of the EGF family, is synthesized and secreted in the embryonic kidney [53], and also binds to the EGF receptor [54], suggesting the TGF $\alpha / E G F R$ complex may regulate the relative amounts of stroma and nephrons in the developing kidney.

\section{Role of the Interstitium in the Adult Kidney}

Stroma, or the interstitium as it is more commonly referred to in the adult kidney, plays a critical role in many functions of the adult kidney. Cells of the interstitium shape the ECM and also play a role in the production of regulatory substances and in immune response [55]. The small amount of interstitium in the cortex is involved in fluid and solute exchange between tubules and the peritubular capillary network. Furthermore, cortical fibroblasts produce erythropoietin [56]. Medullary interstitial cells are involved in the synthesis and secretion of vasodepressor substances and thereby regulate the actions of the renin-angiotensin system [57].

\section{Conclusion}

In recent years, our understanding of the role of the renal stroma in metanephric development has evolved from 'just a supportive framework' to an active and important regulator of both nephrogenesis and ureteric branching morphogenesis. Unfortunately however, unanswered questions and confusion remain regarding the origin, precise location and role of the renal stroma in the developing metanephros.

For many years, renal stromal cells were thought to represent a developmental pathway for blastemal cells which were not induced to undergo nephrogenesis. In recent years, many researchers have described renal stromal cells as being derived from 'pre-determined' blastemal cells of the nephrogenic zone. However, stromal cells do not express the characteristic markers of metanephric mesenchymal cells. Rather, renal stromal cells express many molecules usually associated with neurons. To date, however, there is no direct experimental evidence for the neural crest origin of all or any renal stromal cells. The question of the origin of renal stromal cells will only be answered once we know the origin and lineage relationships of all cells in the metanephric mesenchyme.

The difficulty in defining the renal stroma has contributed to the confusion of researchers in the field. Are all non-ureteric bud, non-cap, non-blastemal cells, cells of the renal stroma? This question is difficult to answer, considering that cells of the primary interstitium differentiate to form the cortical and medullary stroma, which then differentiate to form the interstitium of the adult kidney. These cells seem to be responding to changes in their local environments, making it difficult to put specific locations to particular stromal cell types.

It is now clear that there are not only different types of renal stroma and renal stromal cells, but that these cells play much more than a physically supportive role in kidney development. Stromal cells play important roles in nephrogenesis through regulation of MET, by negatively regulating signals that antagonize tubulogenesis. Further- e6

Nephron Exp Nephrol 2005;99:e1-e8
Cullen-McEwen/Caruana/Bertram 
more, the renal stroma also partially regulates branching morphogenesis through regulating expression of RET in the ureteric bud. However, although much has been learnt about some of the molecules involved in the regulation of these processes, details of the precise molecular mechanisms are still unknown.

\section{Acknowledgments}

The authors thank Prof. Daine Alcorn for helpful discussions and critically reviewing the manuscript.

\section{References}

1 Clark AT, Bertram JF: Molecular regulation of nephron endowment. Am J Physiol Renal Physiol 1999;276 (pt 2):F485-497.

$\checkmark 2$ Clark AT, Bertram JF: Advances in renal development. Curr Opin Nephrol Hypertens 2000;9:247-251.

$\checkmark 3$ Burrow CR: Regulatory molecules in kidney development. Pediatr Nephrol 2000;14:240253.

$\checkmark 4$ Kuure S, Vuolteenaho R, Vainio S: Kidney morphogenesis: Cellular and molecular regulation. Mech Dev 2000;92:31-45.

$\checkmark 5$ Pohl M, Stuart RO, Sakurai H, Nigam SK: Branching morphogenesis during kidney development. Annu Rev Physiol 2000;62:595-620.

$\checkmark 6$ Davies JA, Fisher CE: Genes and proteins in renal development. Exp Nephrol 2002;10:102113.

7 Bard JB: Growth and death in the developing mammalian kidney: Signals, receptors and conversations. Bioessays 2002;24:72-82.

8 Larsen WJ: Human Embryology. New York, Churchill Livingstone, 1993.

9 Sariola H: Fates of the metanephric mesenchyme; in Vice P, Woolf A, Bard J (eds): The Kidney: From Normal Development to Congenital Disease. London, Academic Press, 2003, pp 181-193.

10 Grobstein C: Inductive interaction in the development of the mouse metanephros. J Exp Zool 1955;130:319-340.

11 Grobstein C: Mechanisms of organogenetic tissue interaction. Natl Cancer Inst Monogr 1967; 26:107-119.

12 Ekblom P, Weller A: Ontogeny of tubulointerstitial cells. Kidney Int 1991;39:394-400.

13 Al-Awqati Q, Oliver JA: Stem cells in the kidney. Kidney Int 2002;61:387-395.

14 Bard JB: The metanephros; in Vice P, Woolf A, Bard J (eds): The Kidney: From Normal Development to Congenital Disease. London, Academic Press, 2003, pp 181-193.

15 Sariola H, Holm K, Henke-Fahle S: Early innervation of the metanephric kidney. Development. 1988;104:589-599.

-16 Sainio K, Nonclercq D, Saarma M, Palgi J, Saxen L, Sariola H: Neuronal characteristics in embryonic renal stroma. Int J Dev Biol 1994; 38:77-84.

$\checkmark 17$ Sariola H, Aufderheide E, Bernhard H, HenkeFahle S, Dippold W, Ekblom P: Antibodies to cell surface ganglioside GD3 perturb inductive epithelial-mesenchymal interactions. Cell 1988;54:235-245.
18 Le Douarin NM, Teillet MA: Experimental analysis of the migration and differentiation of neuroblasts of the autonomic nervous system and of neurectodermal mesenchymal derivatives, using a biological cell marking technique. Dev Biol 1974;41:162-184.

19 Bronner-Fraser M, Fraser SE: Cell lineage analysis reveals multipotency of some avian neural crest cells. Nature 1988;335:161-164.

20 Hatini V, Huh SO, Herzlinger D, Soares VC, Lai E: Essential role of stromal mesenchyme in kidney morphogenesis revealed by targeted disruption of winged helix transcription factor BF-2. Genes Dev 1996;10:1467-1478.

21 Alcorn D, Maric C, McCausland J: Development of the renal interstitium. Pediatr Nephrol 1999;13:347-354.

-22 Kanwar YS, Carone FA, Kumar A, Wada J, Ota K, Wallner EI: Role of extracellular matrix, growth factors and proto-oncogenes in metanephric development. Kidney Int 1997;52: 589-606.

23 Maric C, Ryan GB, Alcorn D: Embryonic and postnatal development of the rat renal interstitium. Anat Embryol (Berl) 1997;195:503-514.

24 Marxer-Meier A, Hegyi I, Loffing J, Kaissling B: Postnatal maturation of renal cortical peritubular fibroblasts in the rat. Anat Embryol (Berl) 1998;197:143-153.

25 Mendelsohn C, Batourina E, Fung S, Gilbert T, Dodd J: Stromal cells mediate retinoid-dependent functions essential for renal development. Development 1999;126:1139-1148.

26 Pfaller W: Structure function correlation on rat kidney. Quantitative correlation of structure and function in the normal and injured rat kidney. Adv Anat Embryol Cell Biol 1982;70:1106.

27 Tisher C, Madsen KM: Anatomy of the kidney; in Brenner \& Rector's The Kidney, ed 6. London, Saunders, 1999.

28 Lemley KV, Kriz W: Anatomy of the renal interstitium. Kidney Int 1991;39:370-381.

29 Klein G, Ekblom P: Extracellular matrix composition during kidney development. Contrib Nephrol 1990;80:17-31.

30 Furness PN: Extracellular matrix and the kidney. J Clin Pathol 1996;49:355-359.

31 Batourina E, Gim S, Bello N, Shy M, ClagettDame M, Srinivas S, Costantini F, Mendelsohn C: Vitamin A controls epithelial/mesenchymal interactions through Ret expression. Nat Genet 2001;27:74-78.
32 Li E, Sucov HM, Lee KF, Evans RM, Jaenisch $\mathrm{R}$ : Normal development and growth of mice carrying a targeted disruption of the alpha 1 retinoic acid receptor gene. Proc Natl Acad Sci USA 1993;90:1590-1594.

33 Lufkin T, Lohnes D, Mark M, Dierich A, Gorry P, Gaub MP, Lemeur M, Chambon P: High postnatal lethality and testis degeneration in retinoic acid receptor alpha mutant mice. Proc Natl Acad Sci USA 1993;90:7225-7229.

34 Mendelsohn C, Mark M, Dolle P, Dierich A, Gaub MP, Krust A, Lampron C, Chambon P: Retinoic acid receptor beta 2 (RAR beta 2) null mutant mice appear normal. Dev Biol 1994; 166:246-258.

35 Ghyselinck NB, Dupe V, Dierich A, Messaddeq N, Garnier JM, Rochette-Egly C, Chambon P, Mark M: Role of the retinoic acid receptor beta (RAR beta) during mouse development. Int J Dev Biol 1997;41:425-447.

36 Qiao J, Uzzo R, Obara-Ishihara T, Degenstein L, Fuchs E, Herzlinger D: FGF-7 modulates ureteric bud growth and nephron number in the developing kidney. Development 1999; 126:547-554.

37 Mason IJ, Fuller-Pace F, Smith R, Dickson C: FGF-7 keratinocyte growth factor. Expression during mouse development suggests roles in myogenesis, forebrain regionalisation and epithelial mesenchymal interactions. Mech Dev 1994;45:15-30.

38 Finch PW, Cunha GR, Rubin JS, Wong J, Ron D: Pattern of keratinocyte growth factor and keratinocyte growth factor receptor expression during mouse fetal development suggests a role in mediating morphogenetic mesenchymal-epithelial interactions. Dev Dyn 1995;203:223240.

39 Cancilla B, Ford-Perriss MD, Bertram JF: Expression and localization of fibroblast growth factors and fibroblast growth factor receptors in the developing rat kidney. Kidney Int 1999; 56:2025-2039.

40 Raatikainen-Ahokas A, Hytonen M, Tenhunen A, Sainio K, Sariola H: Bmp-4 affects the differentiation of metanephric mesenchyme and reveals an early anterior-posterior axis of the embryonic kidney. Dev Dyn 2000;217:146158.

41 Schnabel CA, Selleri L, Cleary ML: Pbx1 is essential for adrenal development and urogenital differentiation. Genesis 2003;37:123-130. 
42 Quaggin SE, Schwartz L, Cui S, Igarashi P, Deimling J, Post M, Rossant J: The basichelix-loop-helix protein Pod1 is critically important for kidney and lung organogenesis. Development 1999;126:5771-5783.

43 Cui S, Schwartz L, Quaggin SE: Pod1 is required in stromal cells for glomerulogenesis. Dev Dyn 2003;226:512-522.

44 Yang J, Blum A, Novak T, Levinson R, Lai E, Barasch J: An epithelial precursor is regulated by the ureteric bud and by the renal stroma. Dev Biol 2002;246:296-310.

-45 Barasch J, Yang J, Ware CB, Taga T, Yoshida $\mathrm{K}$, Erdjument-Bromage $\mathrm{H}$, Tempst P, Parravicini E, Malach S, Aranoff T, Oliver JA: Mesenchymal to epithelial conversion in rat metanephros is induced by LIF. Cell 1999;99:377386.

46 Lyons KM, Hogan BLM, Robertson EJ: Colocalization of Bmp 7 and Bmp 2 RNAs suggests that these factors cooperatively mediate tissue interactions during murine development. Mech Dev 1995;50:71-83.
47 Vukicevic S, Kopp JB, Luyten FP, Sampath TK: Induction of nephrogenic mesenchyme by osteogenic protein 1 (bone morphogenetic protein 7). Proc Natl Acad Sci USA 1996;93: 9021-9026.

48 Godin RE, Takaesu NT, Robertson EJ, Dudley AT: Regulation of BMP7 expression during kidney development. Development 1998;125: 3473-3482.

49 Weller A, Sorokin L, Illgen EM, Ekblom P: Development and growth of mouse embryonic kidney in organ culture and modulation of development by soluble growth factor. Dev Biol 1991;144:248-261.

50 Nexo E, Kryger-Baggesen N: The receptor for epidermal growth factor is present in human fetal kidney, liver and lung. Regul Pept 1989; 26:1-8.

51 Perheentupa J, Laksmanahan J, Fisher DA: Urine and kidney epidermal growth factor: Ontogeny and sex difference in the mouse. Pediatr Res 1985;19:428-432.

52 Popliker M, Shatz A, Aviva A, Ullrich A, Schlessinger J, Webb CG: Onset of endogenous synthesis of epidermal growth factor in neonatal mice. Dev Biol 1987;119:38-44.
53 Rogers SA, Ryan G, Hammerman MR: Metanephric transforming growth factor-alpha is required for renal organogenesis in vitro. Am J Physiol 1992;262 (pt 2):F533-539.

54 Bernardini N, Bianchi F, Lupetti M, Dolfi A: Immunohistochemical localization of the epidermal growth factor, transforming growth factor alpha, and their receptor in the human mesonephros and metanephros. Dev Dyn 1996;206:231-238.

55 Fine LG, Norman JT, Ong A: Cell to cell crosstalk in the pathogenesis of renal interstitial fibrosis. Kidney Int 1995;47(suppl 49):S4850.

56 Bachmann S, Le Hir M, Eckardt Ku: Co-localization of erythropoietin mRNA and ecto-5' nucleotidase immunoreactivity in peritubular cells of rat renal cortex indicates that fibroblasts produce erythropoietin. J Histochem Cytochem 1993;41:335-341.

57 Thomas CJ, Woods RL, Evans RG, Alcorn D, Christy IJ, Anderson WP: Evidence for a renomedullary vasodepressor hormone. Clin Exp Pharmacol Physiol 1996;23:777-785. 